\title{
Unraveling Cyclic Deformation Mechanisms of a Rolled Magnesium Alloy Using In-situ Neutron Diffraction
}

\author{
Wei $\mathrm{Wu}^{1}$, Peter K. Liaw ${ }^{2}$, and $\mathrm{Ke} \mathrm{An}^{1, *}$
}

1. Chemical and Engineering Materials Division, Oak Ridge National Laboratory, Oak Ridge, TN 37831, USA

2. Department of Materials Science and Engineering, The University of Tennessee, Knoxville, TN 37996, USA

\section{*Corresponding author}

Ke An, Ph.D.

Lead Scientist

Chemical and Engineering Materials Division

Oak Ridge National Laboratory

1 Bethel Valley Rd

Oak Ridge

TN 37831, USA

Office: $+1-865-919-5226$

Fax: +1-865-574-6080

E-mail: kean@ornl.gov 


\begin{abstract}
:
In the current study, the deformation mechanisms of a rolled magnesium alloy were investigated under cyclic loading using the real-time in-situ neutron diffraction under a continuous-loading condition. The relationship between the macroscopic cyclic deformation behavior and the microscopic response at the grain level has been established. The neutron diffraction results indicate that more and more grains are involved in the twinning and detwinning deformation process with the increase of fatigue cycles. The residual twins appear in the early fatigue life, which is responsible for the cyclic hardening behavior. The asymmetric shape of the hysteresis loop is attributed to the early exhaustion of the detwinning process during compression, which leads to the activation of dislocation slips and rapid strain hardening. The critical resolved shear stress for the activation of tensile twinning closely depends on the residual strain developed during cyclic loading. In the cycle before the sample fractured, the dislocation slips became active in tension, although the sample was not fully twinned. The increased dislocation density leads to the rise of the stress concentration at weak spots, which is believed to be the main reason for the fatigue failure. The deformation history greatly influences the deformation mechanisms of hexagonal close-packed structured magnesium alloy during cyclic loading.
\end{abstract}

Keyword: Magnesium alloy; Cyclic loading; Deformation mechanisms; Neutron diffraction; Twinning 


\section{Introduction}

Magnesium (Mg) alloys drew great attentions to automobile and air-craft industries over last two decades due to the integration of low-density, high specific strength, excellent thermal conductivity, and extraordinary damping capacity [1-4]. Mg alloys are the lightest metal commercially available that can be used as structural materials. For applications as the loadbearing components, it is vital to understand the fatigue behavior of $\mathrm{Mg}$ alloys for the reliability and durability concerns. In cast $\mathrm{Mg}$ alloys, defects, such as casting porosity and inclusion, which commonly act as crack sources, are harmful to fatigue properties, and may assist fatigue-crack initiation, reduce lifetimes, and decrease cyclic strength [5, 6]. On the contrary, wrought $\mathrm{Mg}$ alloys are basically defect-free, and, thus, exhibit better fatigue properties than casting alloys [79]. Moreover, the study on the cyclic behavior of wrought $\mathrm{Mg}$ alloys may shed light on the intrinsic fatigue mechanisms of $\mathrm{Mg}$ alloys [10]. Therefore, mechanistic understanding on the cyclic-deformation behavior can facilitate the practical applications of wrought $\mathrm{Mg}$ alloys as structural materials.

Five independent slip systems are necessary in order to achieve arbitrary homogenous deformation for polycrystalline materials. $\mathrm{Mg}$ has a hexagonal close-packed (HCP) structure with a limited number of slip systems. In principle, $\mathrm{Mg}$ has five independent slip systems, including two $(00.2)<11.0>$ slip (or basal < a $>$ slip), one $(10.0)<11.0>$ slip (or prismatic $<$ a $>$ slip), one $(10.1)<11.0>$ slip (or pyramidal <a $>$ slip), and one $(11.2)<11.3>$ slip (or pyramidal $<\mathrm{c}+\mathrm{a}>$ slip) [11-13]. The pyramidal $<\mathrm{c}+\mathrm{a}>$ slip is the only one that can provide strain along the c-axis. However, the pyramidal $<\mathrm{c}+\mathrm{a}>$ slip is very difficult to be activated at room temperature, due to the high critical resolved shear stress [14]. Thus, deformation twinning plays as an addition to the slip systems to satisfy the von Mises yield criterion, but it can only offer a limited amount of 
strain. It has been recognized that the tension-compression asymmetry of wrought $\mathrm{Mg}$ alloys results from the unidirectional nature of the deformation twinning $[12,15]$.

Because the c/a ratio of the HCP-structured $\mathrm{Mg}$ is 1.624 , which is less than the ideal hard-sphere value, $\sqrt{3}$, the most prominent twinning system is $\{10.2\}<10.1>$ tensile twinning (or extension twinning), which provides a tensile strain along the c-axis $[12,13,15,16]$. The $\{10.2\}<10.1>$ tensile twinning leads to a sudden reorientation of the matrix lattice approximately $86.3^{\circ}$ by a tensile stress along the $\mathrm{c}$-axis or a compressive stress perpendicular to the $\mathrm{c}$-axis $[17$, 18]. After tensile twinning, the detwinning process could be easily activated by a stress reverse. The alternate twinning and detwinning process was often reported in the previous studies during strain-path changes and/or cyclic loading in the highly-textured wrought Mg alloys [17-22].

Comprehensive studies have been concentrated on the effects of strain amplitude [23-25], mean stress [26-28], strain ratio [8, 9, 28, 29], strain rate [29], microstructure [8, 30, 31], grain size [32, 33], rare earth elements [34, 35], hysteresis energy [26], heat-treatment [36], temperature [5], environment [37, 38], and initial texture [39-42] on the fully-reversed straincontrolled low-cycle fatigue behavior of the wrought Mg alloys. Moreover, in the last decade, progress has been made in theoretical modeling to predict the slip, twinning, and detwinning behavior in the HCP-structured material during strain-path changes and cyclic loading [11, 22, 39, 43-47]. Overall, the low-cycle fatigue life of the wrought Mg alloys increases with the decrease of strain amplitude, strain ratio, and mean stress, as well as the increase of strain rate. The previous fatigue studies $[7,9,23,39,40,48]$ demonstrated that the strain-life curves can be described well by the Basquin and Manson-Coffin equations, as well as the Holloman relation. Recently, it has been found that a clear kink point around a total strain of 0.005 can be observed from the strain amplitude - fatigue life curve in the extruded AZ61 and ZK60 Mg alloys, 
indicating that the dislocation and twinning-detwinning deformation modes were dominant below and above the 0.005 total strain, respectively [28, 30, 49]. Subsequently, the twinning and detwinning processes usually involved in the fully-reversed strain-controlled low-cycle fatigue at a relatively large total strain $(>0.005)$, which has been proven experimentally by optical microscopy [8], in-situ electron backscatter diffraction (EBSD) [50], in-situ transmission electron microscopy (TEM) [21, 51], and in-situ neutron diffraction measurements [17, 18, 39, 52, 53]. In general, the alternate twinning and detwinning process during cyclic loading is thought to be the main reason for the strong tension-compression asymmetry of the hysteresis loops. However, in the prior in-situ experiments, discontinuous step-loading methods were commonly employed, which contains only a limited number of measurements. Usually, a few data points (at peak strain, zero stress, and zero strain) per fatigue cycle were presented in the previous research $[17,18,39,52]$. Certainly, these limited data points are representative on the hysteresis loop in each fatigue cycle, but the information missing between two measurements is inevitable. For example, in one cycle how the twinning/detwinning behavior evolves and when the transition of twinning/detwinning to dislocation deformation occurs, etc., are not clearly represented by those scarce measurements, and most importantly how those behaviors evolve during fatigue cycling before the material fails are not answered clearly by the previous studies.

In this investigation, these above questions will be addressed by the state-of-the-art realtime in-situ neutron diffraction. Previously, we have reported the effects of strain-path changes and pre-deformation history on the deformation dynamics and mechanisms of a rolled AZ31B Mg alloy investigated by the real-time in-situ neutron diffraction under a continuous loading condition $[20,54]$. In the present research, the influence of cyclic loading on plastic deformation, and twinning and detwinning behavior, including the residual-twin evolution during the fully- 
reversed low-cycle fatigue are presented here in-depth by employing the same experimental method. 


\section{Experimental}

\subsection{Experimental materials}

A commercial rolled AZ31B Mg alloy plate [chemical composition: 3 weight percent (w.t.\%) $\mathrm{Al}, 1$ w.t.\% $\mathrm{Zn}$, and $\mathrm{Mg}$ as balance] in $\mathrm{H} 24$ temper (strain hardened and partially annealed) was selected for the current research, which contains a typical rolling texture with the crystal c-axis parallel to the normal direction (ND) and perpendicular to the rolling direction (RD) of the rolling plate [55]. The thickness of the rolled plate is $76 \mathrm{~mm}$, which facilitate the machining of low-cycle fatigue samples along the through-thickness direction (or ND). Dogbone cylindrical low-cycle fatigue specimens with an 8-mm diameter and 16-mm gage length were fabricated, according to the American Society for Testing and Materials (ASTM) Standard E606-04. After sample machining, all the specimens were annealed at $345{ }^{\circ} \mathrm{C}$ for 2 hours to relieve the existing residual stress. The average grain size of the annealed samples is approximately $40 \mu \mathrm{m}$.

\subsection{Low-cycle fatigue experiment}

The fully-reversed strain-controlled low-cycle fatigue experiment was conducted at a total strain amplitude, $+/-0.02$, at room temperature with triangular loading waveforms. The fatigue cycles, $1^{\text {st }}, 2^{\text {nd }}, 5^{\text {th }}, 10^{\text {th }}, 20^{\text {th }}, 50^{\text {th }}$, and $70^{\text {th }}$ cycles, were selected for the real-time in-situ neutron diffraction measurements before the sample failed in the $71^{\text {st }}$ fatigue cycle. The strain rate in the selected cycles was relatively low for the real-time in-situ neutron diffraction measurements. Specifically, the strain rate in the $1^{\text {st }}$ cycle was $6.8 \times 10^{-6} \mathrm{~s}^{-1}$, in the $2^{\text {nd }}$ cycle was $5.4 \times 10^{-6} \mathrm{~s}^{-1}$, and in the other chosen cycles was $8.3 \times 10^{-6} \mathrm{~s}^{-1}$. Except the fatigue cycles chosen for the neutron diffraction measurements, the frequency in the rest was $1 \mathrm{~Hz}$ with a corresponding strain rate of $8 \times 10^{-2} \mathrm{~s}^{-1}$. 


\subsection{Real-time in-situ neutron diffraction measurements}

The in-situ neutron diffraction measurements were performed at the VULCAN Engineering Materials Diffractometer [56-58], the Spallation Neutron Source (SNS), Oak Ridge National Laboratory (ORNL) during the fully-reversed low-cycle fatigue under a continuousloading condition. Due to the high neutron flux at the VULCAN instrument at SNS, the real-time in-situ neutron diffraction measurements have been successfully employed in a number of studies [20, 54, 59-62]. The experimental setup for the $\mathrm{Mg}$ alloys neutron diffraction measurements under mechanical loading is described in detail elsewhere [20, 54]. In the following, a brief description is provided. The sample was mounted horizontally to the VULCAN loadframe, with the axial direction parallel to ND and the horizontal-radial direction along RD. The angle between the incoming neutron beam and specimen was $45^{\circ}$. The two stationary detector banks are located at $\pm 90^{\circ}$ to the incoming beam. In this setup, two complete diffraction patterns [the axial (ND) and horizontal-radial (RD) directions with diffraction vectors parallel $\left(Q_{\|}\right)$and perpendicular $(\mathrm{Q} \perp)$, respectively] were recorded simultaneously during cyclic loading. The neutron beam size was defined as $5 \mathrm{~mm}$ (horizontal) x $5 \mathrm{~mm}$ (vertical), and the 5$\mathrm{mm}$ receiving collimators were selected, leading to a neutron gauge volume of total $125 \mathrm{~mm}^{3}$. Considering that the average grain size was approximately $40 \mu \mathrm{m}$, the bulk-averaged information with a brilliant counting statistic of a great number of grains was attained. The neutron diffraction measurements were conducted under the high intensity mode with a chopper speed of $20 \mathrm{~Hz}$, resulting in the $\mathrm{d}$-spacing range from 0.5 to 3.5 angstroms.

The neutron diffraction data were recorded consecutively during the low-cycle fatigue experiment. After the measurements, the data reduction was performed, using the event-based data reduction software, VULCAN Data Reduction and Interactive Visualization softwarE 
(VDRIVE) [63]. The neutron diffraction data can be chopped into the shortest attainable time bins. In the current study, the time interval of two minutes was chosen, based on both the quality and the statistics of diffraction patterns for data fitting. The loadframe information (i.e., stress and strain) was averaged over two minutes and synchronized with the chopped neutron diffraction patterns. The neutron diffraction peak intensities of different $h \mathrm{kls}$ were normalized to the incoming beam energy after the background subtraction to eliminate the neutron beam fluctuation during measurements. The counting time for the un-deformed sample was 10 minutes, which is longer than 2 minutes of the chopped data, in order to reduce the propagated $d_{0}^{h k l}$ (dspacing of certain $h k l$ before deformation) statistic error.

The two-dimensional (2-D) and three-dimensional (3-D) visualization of the real-time insitu neutron diffraction results of the measured $d$ range for both the axial and radial directions during low-cycle fatigue are presented in Fig 1. The deformation dynamics in each selected fatigue cycles, including the simultaneous evolution of d-spacing, diffraction peak intensity, and diffraction peak width of certain $h k l s$ in both the axial and radial directions, are illustrated. In the axial direction, as presented in Fig. 1(a), the diffraction peak intensity of the (10.0) grains experienced an appearing-disappearing sequence during tension-compression in each fatigue cycle, corresponding to the twinning-detwinning deformation. Meanwhile, the d-spacing of the (00.2) grains underwent a repeatedly increasing-decreasing sequence in Fig. 1(a), due to cyclic loading. In Fig. 1(c), the diffraction peak intensity of the (00.2) grains in the axial direction exhibits a repetitively decreasing-increasing sequence, designating the twinning-detwinning behavior during cyclic loading. The opposite trend can be perceived in the radial direction, as displayed in Figs. 1(b) and (d). More detailed descriptions and discussions will be provided in the results and discussion sections. 


\section{Results}

3.1 Macroscopic low-cycle fatigue behavior

The hysteresis loops of the selected fatigue cycles for the real-time in-situ neuron diffraction measurement are plotted in different colors, as shown in Fig. 2(a). The Roman numerals, I, II, III, IV, V, VI, and VII, marked in Fig. 2(a), stand for the maximum strain (+ 0.02), zero stress on the tensile strain side, zero strain during compression, minimum strain $(-0.02)$, zero stress on the compressive strain side, zero strain during tension, and maximum strain, respectively. The asymmetric shape of the hysteresis loops was observed through the entire fatigue life (the sample failed at the $71^{\text {st }}$ cycle). In general, the stress-strain curve on the tension side demonstrated a typical concave-down shape, while the compression side exhibited a concave-up shape. However, in the $1^{\text {st }}$ cycle (black line), a sigmoidal shape with the concavedown first and followed by concave-up was noticed during compression, which was different from the other fatigue cycles. For the initial tension in the $1^{\text {st }}$ cycle [marked on the Fig. 2(a)], the $0.2 \%$ proof stress was much lower than the following fatigue cycles on the tension side. Moreover, the entire stress-strain curve of the initial tension was below that of the other fatigue cycles during tension. During compression in the $1^{\text {st }}$ cycle, the stress-strain curve exhibited stable strain hardening, followed by sudden strain hardening, and then another constant strain hardening, while the stress-strain curve in the other fatigue cycles displayed steady strain hardening, followed by rapid strain hardening. The mechanical behavior during reverse tension in the $1^{\text {st }}$ fatigue cycle is comparable with that in the following fatigue cycles on the tension side.

The peak stresses at the maximum and minimum strains [black solid square symbols, corresponding to I and IV in Fig. 2(a), respectively], the compressive and tensile stresses at the zero strain [blue open triangle symbols corresponding to III and VI in Fig. 2(a), respectively], 
and the mean stress (red open circle symbols) variation during low-cycle fatigue are illustrated in Fig. 2(b). The compressive peak stresses were much higher than the tensile peak stresses in each selected fatigue cycle, which led to a compressive mean stress throughout the fatigue life. The apparent strain hardening was distinguished from the tensile peak stress evolution in the first five cycles, followed by a slight strain hardening response. On the other hand, the compressive peak stress was saturated after five fatigue cycles. The consistence of mean stresses during the fatigue tests was perceived. A slight increase was obtained for both the tensile and compressive stresses at the zero strain in the first five cycles. After that, the tensile and compressive stresses at the zero strain reached the maximum values and kept constant. The tensile and compressive stresses at zero strain during low-cycle fatigue are comparable over the entire fatigue life.

The $0.2 \%$ proof stress evolution of the selected fatigue cycles is plotted in Fig. 2(c). The $0.2 \%$ proof stress of the initial tension in the $1^{\text {st }}$ cycle was only $65 \mathrm{MPa}$, which was much lower than the other fatigue cycles. The $0.2 \%$ proof stress during tension kept increasing in the first 5 cycles, and then stayed unchanged. The $0.2 \%$ proof stress during compression increased continuously until the $50^{\text {th }}$ cycle, whereas it remained almost constant between the $50^{\text {th }}$ and $70^{\text {th }}$ cycles. The tensile $0.2 \%$ proof stress was higher than the compressive $0.2 \%$ proof stress in each cycle throughout fatigue testing, which had an opposite trend as the peak stress evolution in Fig. 2(b). It implies that the detwinning process can be more easily activated in compression than the tensile twinning dominated deformation in tension. Moreover, in the early fatigue life, up to the $20^{\text {th }}$ cycle, the differences between tensile and compressive $0.2 \%$ proof stresses decreased persistently, while it stopped changing after the $20^{\text {th }}$ cycle.

The plastic strain on the tension side [II in Fig. 2(a)], the plastic strain on the compression side [V in Fig. 2(a)], and the plastic strain amplitude, $\Delta \varepsilon_{p} / 2$, as a function of fatigue cycles are 
shown in Fig. 2(d). The tensile plastic strains were marginally higher than the compressive plastic strains during low-cycle fatigue. For the plastic strains on the tension side, a slight decrease was observed in the early fatigue life (up to the $5^{\text {th }}$ cycle), and then the tensile plastic strains ceased changing in the following fatigue cycles. The plastic strains on the compression side remained unchanged in the entire fatigue life. Thus, there was little change in the plastic strain amplitude during cyclic loading.

The Kocks-Mecking type plot of work-hardening rates, $\theta$, as a function of net flow stress, $\sigma-\sigma_{0.2}$ (where $\sigma$ and $\sigma_{0.2}$ are the engineering stress and the $0.2 \%$ proof stress, respectively) in each selected fatigue cycles are elucidated in Fig. 3. The hardening rate was calculated, based on the well-known equation below:

$$
\theta=\frac{d \sigma}{d \varepsilon}
$$

where $\theta$ is the work-hardening rate, $\sigma$ is the stress, and $\varepsilon$ is the strain. Based on the workhardening rate changes in Fig. 3, the plastic deformation was separated into several regions, marked as letters and numbers. The black and purple dash-dot lines represent the work-hardening rate variation in the $1^{\text {st }}$ and $70^{\text {th }}$ cycle, respectively. During tension in the $1^{\text {st }}$ cycle, the workhardening rate decreased dramatically until the net flow stress reached approximately $3 \mathrm{MPa}$, and then the work-hardening rate increased slightly as shown in Fig. 3(a). The similar phenomenon can be observed in the following fatigue cycles, except that the inflection point moved to the higher net flow stress side with the increase of fatigue cycles. It can be noticed that in the $50^{\text {th }}$ and $70^{\text {th }}$ cycles, a sudden increase of the work-hardening rate appeared, when the net flow stress passed $34 \mathrm{MPa}$. During compression in the $1^{\text {st }}$ fatigue cycle, the work-hardening rate stayed almost constant below - $22 \mathrm{MPa}$, and enhanced significantly until - $86 \mathrm{MPa}$, and then reduced 
rapidly, as demonstrated in Fig. 3(b). The overall trend of the work-hardening rate evolution in the $2^{\text {nd }}$ fatigue cycle during compression was analogous to the $1^{\text {st }}$ cycle. However, the whole curve of the $2^{\text {nd }}$ cycle shifted to the right-hand side, and the maximum work-hardening rate in the $2^{\text {nd }}$ cycle increased to $18663 \mathrm{MPa}$, which is higher than that in the $1^{\text {st }}$ cycle, $16465 \mathrm{MPa}$. Moreover, a work-hardening rate decrease region was observed until the net flow stress reached - $19 \mathrm{MPa}$ in the $2^{\text {nd }}$ cycle, which was not perceived in the $1^{\text {st }}$ cycle. Furthermore, the stress range of the work-hardening rate increase was expanded and that of the work-hardening rate decrease was shortened in the $2^{\text {nd }}$ cycle, comparing with the $1^{\text {st }}$ cycle. Since the $5^{\text {th }}$ cycle until the sample failure, the curves of work-hardening rate vs. net flow stress almost overlapped. The workhardening rate decreased obviously when the net flow stress approached approximately - 25 $\mathrm{MPa}$, followed by a stable work-hardening rate region up to - $50 \mathrm{MPa}$, and then the workhardening rate increased promptly. It is worth mentioning that above - $90 \mathrm{MPa}$, the workhardening rate decreased with the increase of fatigue cycles at the same stress level from the $5^{\text {th }}$ to $70^{\text {th }}$ cycle. In other words, the rate of the work-hardening rate increase decelerated after the $5^{\text {th }}$ cycle.

\subsection{Microscopic response by neutron diffraction}

The diffraction peak intensity evolutions of certain $h k l s$ in both the axial and radial directions in the selected fatigue cycles are presented in Fig. 4(a) and (b), respectively. As previously mentioned, the tensile twinning-dominated deformation leads to a sudden reorientation of grains approximately $86^{\circ}$, owing to the HCP structure of magnesium. Consequently, when the tensile twinning is activated (tension along ND in the present study), the diffraction peak intensity of the (00.2) grains decreases, which is the majority in the axial direction before deformation, and the (10.0) diffraction peak intensity increases simultaneously 
on the same detector bank. While in the radial direction, the opposite trend can be anticipated that the (00.2) diffraction peak intensity increases and the (10.0) peak intensity decreases. The alternating trend of the increase-decrease or decrease-increase sequence was distinguished from the diffraction peak intensities of the (10.0), (00.2), and (11.0) grains in both the axial and radial directions during fully-reversed low-cycle fatigue, as demonstrated Fig. 4(a) and (b). The simultaneous variation of the diffraction peak intensities of the (10.0) and (00.2) grains was also found in the same detector bank. It manifests that the alternative twinning and detwinning deformations occurred during the tension-compression loading sequence in each fatigue cycle. It should be pointed out that, in Fig. 4(a), the decrease of the diffraction peak intensity in the (00.2) grains during initial tension was apparently less than during the $2^{\text {nd }}$ tension in the $1^{\text {st }}$ fatigue cycle, which was caused by different tension strains. As shown in Fig. 2(a) in the $1^{\text {st }}$ fatigue cycle, the tensile strain in the initial tension was 0.02 , while it became approximately 0.033 in the $2^{\text {nd }}$ tension (from -0.013 to +0.02 strain).

It is noticed that the diffraction peak intensity of the (00.2) grains in the axial direction (red solid symbols) at the maximum strain, +0.02 , decreased progressively in Fig. 4(a) [or increased in Fig. 4(b)] with the increase of fatigue cycles (marked as the purple dashed line), suggesting more and more (00.2) grains involved in the tensile twinning deformation. Moreover, in the $1^{\text {st }}, 2^{\text {nd }}, 5^{\text {th }}$, and $10^{\text {th }}$ fatigue cycles, the diffraction peak intensity of the $(00.2)$ grains was fully recovered during compression, as shown in Fig. 4. However, after the $10^{\text {th }}$ cycle, the diffraction peak intensity of the (00.2) grains at the minimum strain, -0.02 , gradually decreased in Fig. 4(a) [or increased in Fig. 4(b)] with fatigue cycles (marked as the green-dashed line). It implies that in the first 10 fatigue cycles, the twins from tension were fully detwinned after the compression in each fatigue cycle, while since the $20^{\text {th }}$ fatigue cycle, the accumulated residual 
twins appeared.

The diffraction peak intensity evolution of certain $h k l s$ in the axial direction during cyclic loading is demonstrated in Fig. 5. In the previous studies [20, 54], the various dominant deformation modes in different stress ranges were labeled during strain-path changes, based on the work-hardening rate changes and the simultaneous diffraction peak intensity variations of (10.0) and (00.2) grains. In the current study, the same method was employed to correlate the different deformation modes with the work-hardening rate changes. The letters and numbers in Fig. 5 are adopted from Fig. 3. In the $1^{\text {st }}$ cycle, the diffraction peak intensity of the (00.2) grains in the axial direction kept unchanged until the $0.2 \%$ proof stress, followed by a gradual drop in the stress range "a" (a work-hardening rate decrease region), as illustrated in Fig. 5(a). In the stress range "b" (a work-hardening rate slight increase region), the (00.2) peak intensity decreased promptly, which indicates an intensive tensile twinning dominated deformation mode. The opposite trend can be observed in the (10.0) grains in the axial direction, as displayed in Fig. 5(b), that the diffraction peak intensity of the (10.0) grains increased slightly in the stress range "a", and then increased swiftly in the stress range "b". Besides the aforementioned diffraction peak intensity changes during tension, the decrease of the diffraction peak intensity of the $(00.2)$ grains in the axial direction decelerated in the stress range " $C$ " (a work-hardening rate sudden increase region) in the $70^{\text {th }}$ cycle (the purple dash-dot line), as shown in Fig. 5(a). Overall, the tensile twinning dominated deformation mode prevailed in the plastic deformation region during tension in all of the fatigue cycles.

During compression in the $1^{\text {st }}$ cycle, the (00.2) [or the (10.0)] peak intensity enhanced (or decrease) rapidly in the stress range 1 (a constant work-hardening rate region), as displayed in Fig. 5(c) [or (d)]. When the stress was in the stress range 2 (a work-hardening rate increase 
region), the increase of diffraction peak intensity of the (00.2) [or (10.0)] grains decelerated. When the sample was further compressed, the (00.2) [or (10.0)] peak intensity ceased changing in the stress range 3 (a work-hardening rate decrease region). Consequently, three distinct plastic deformation modes, detwinning, transition from detwinning to dislocation, and dislocation slip, were distinguished in the $1^{\text {st }}$ fatigue cycle, corresponding to stress ranges 1,2 , and 3 , respectively. The deformation mechanisms during compression in the $2^{\text {nd }}$ cycle are as same as the $1^{\text {st }}$ cycle. From the $5^{\text {th }}$ to $70^{\text {th }}$ cycle, the diffraction peak intensity of the (00.2) [or (10.0)] grains increased (or decreased) relatively slow in the stress range (1) (a work-hardening rate decrease region), as illustrated in Fig. 5 (c) [or (d)], comparing with the stress range (2) (a constant work-hardening rate region), which indicates that the detwinning dominated deformation is less intensive in the stress range (1) than (2). In the stress range (3) (a work-hardening rate increase region), the diffraction peak intensities of (00.2) [or (10.0)] grains increased (or decrease) slowly, which implies that the detwinning process was still active. The plastic deformation in the stress range (1) and (2) were governed by the detwinning process, and that in the stress range (3) was ruled over by the transition from detwinning to dislocation dominated deformation. In summary, it is found that the dislocation dominated deformation only existed in the first two cycles during compression, and only detwinning and transition from detwinning to dislocation dominated deformation modes remained with the increase of fatigue cycles. It suggests that the deformation history has a significant impact on deformation modes in compression during cyclic loading. 


\section{Discussion}

\subsection{Low-cycle fatigue behavior}

In the current study, the reason for the asymmetry of the stress-strain hysteresis loop could be understood by the clearly defined deformation modes transitions through the rich information developed from the real-time in-situ neutron diffraction measurements. The stressstrain curve of the $20^{\text {th }}$ fatigue cycle is chosen as an example for the detailed analyses in Fig. 6 (a). Based on the neutron diffraction results, the stress-strain hysteresis loop of the $20^{\text {th }}$ fatigue cycle in Fig. 6(a) can be separated into three regions, including, (One) tensile twinning dominated deformation in tension (filled with the diagonal pattern in red), (Two) detwinning dominated deformation (filled with the diagonal pattern in blue), and (Three) transition from detwinning to dislocation dominated deformation (filled with the diagonal pattern in green) in compression. The dividing point of the abrupt work-hardening rate change (a black dot) during compression is marked in Fig. 6(a). An imaginary line (a black dashed line) is drawn in consonance with the stress-strain flow in the detwinning dominated deformation region. It is obvious that the regions (One) and (Two) are symmetric in shape to each other. The asymmetry of the stress-strain hysteresis loop results from the region (Three). As demonstrated in Fig. 4(a), the (00.2) grains in the axial direction were never fully twinned during low-cycle fatigue, since the diffraction peak intensities of the (00.2) grains are always higher than zero. In other words, a majority of grains is suitable for tensile twinning, which happens to be the easiest deformation mode in the current loading condition (tension along ND), but only a portion of those grains involves in tensile twinning to achieve the imposed macroscopic strain [0.033 tensile strain in the $20^{\text {th }}$ cycle in Fig. 6(a)]. The detwinning dominated deformation is completed after a compressive strain of -0.027 , as shown in Fig. 6(a). Unlike in the tension region that a large amount of grains 
are suitable for the tensile twinning deformation, the detwinning process only operates in the twinned grains. Apparently, the quantity of twinned grains is not abundant enough to accomplish in the total compressive strain of -0.036 . By the end of the detwinning dominated deformation, the grain orientation is not applicable for detwinning deformation, and the enhanced stress is high enough to trigger the dislocation slip readily in the majority of the grains. Hence, the early exhaustion of the detwinning process in compression is thought to be the main reason for the asymmetric shape of the stress-strain hysteresis loop, since the dislocation slip at a relatively higher stress level is activated in order to accommodate a further imposed compressive strain, about -0.009 , which leads to the rapid strain hardening and stress increase.

The peak stresses (solid squares), $0.2 \%$ proof stresses (open circles), stresses at the dividing points between the detwinning and transition from the detwinning to dislocation dominated deformation region (open triangles), and stresses at the dividing points between the transition and dislocation dominated deformation (open five-pointed stars) as a function of fatigue cycles are illustrated in Fig. 6(b). The stress ranges for the tensile twinning dominated deformation (R1), detwinning dominated deformation (R2), and transition from detwinning to dislocation dominated deformation (R3) are filled with diagonal patterns by red, blue, and green colors, respectively. It is found that the stress range $\mathrm{R} 1$ is identical to the stress range $\mathrm{R} 2$ during cyclic loading. The transition from the detwinning to dislocation dominated deformation region contributes nearly $50 \%$ of the compressive peak stress after the first two cycles. The tensile peak stresses (black solid squares) are comparable to those at the dividing points between the detwinning and the transition region (red open triangles) during compression in each fatigue cycle, except the $1^{\text {st }}$ cycle, in which the tensile peak stress was much higher than those at the dividing points. Thus, it is further confirmed that the rapid strain hardening due to the activation 
of dislocation slips in the transition region is responsible for the asymmetry of the stress-strain hysteresis loop throughout the entire fatigue cycling.

Moreover, it is found that the macroscopic stress ranges R1 and R2 became wider and wider with the increase of fatigue cycles in Fig. 6(b), which suggests that the stress ranges for twinning and detwinning processes expand over strain cycling. Microscopically, more and more grains involved in the twinning and detwinning deformation, based on the diffraction peak intensity evolution in (10.0) and (00.2) grains in Fig. 4, which is responsible for the expansion of the stress ranges R1 and R2. Hence, the relationship between the macroscopic mechanical behavior and microscopic response has been successfully established, using the real-time in-situ neutron diffraction measurement in the current study.

In addition, the residual twins were observed in the early fatigue life (the $20^{\text {th }}$ cycle) and accumulated with fatigue cycles in Fig. 4. The previous researchers [17] stated that the residual twins are in the "hard" orientation and difficult to deform. The residual twins could serve as barriers to obstruct the dislocation motion. Moreover, with the increase of fatigue cycles, the amount of twin boundaries increases, which could serve as the barriers to hinder the dislocation motion. Both of them contribute to the strain hardening behavior over the fatigue cycles.

4.2 Critical resolved shear stress (CRSS) evolution during low-cycle fatigue

It has been widely accepted that the Schmid law [Eq. (2)] is valid to calculate the onset of deformation twinning under a uniaxial loading condition by assuming that the onset of the deformation twinning is governed by the critical resolved shear stress (CRSS) on the twin plane and in the twinning direction $[16-18,64,65]$ :

$$
\tau_{C R S S}=\sigma_{c} \cos \lambda \cos \chi
$$


where $\tau_{\text {CRSS }}$ is the CRSS, $\sigma_{c}$ is the critical applied stress, $\lambda$ and $\chi$ are the angles between the loading axis and twin plane normal and between the loading axis and the twinning direction, respectively. The CRSS was derived according Eq. (2) and is shown as a function of the fatigue cycles in Fig. 7. It is obvious that the CRSS of the initial tension in the $1^{\text {st }}$ fatigue cycle is much lower than in the other fatigue cycles. It manifests that the tensile twinning can be more easily activated in the annealed sample than in the fatigued sample. The residual lattice strain evolution of the (00.2) grains in the axial direction is also presented in Fig. 7. It is clearly that the CRSS variation in different fatigue cycles has an identical trend as the residual lattice strain evolution of the (00.2) grains in the axial direction after the compression. It is believed that the compressive residual strain developed during cyclic loading is responsible for the increase of the CRSS and the postponement of the activation of the tensile twinning dominated deformation in tension.

\subsection{Plastic deformation mechanisms during cyclic loading}

The difference in the microscopic response during compression is accountable for the dissimilar hysteresis loop shape of the stress-strain curve in the $1^{\text {st }}$ cycles. As demonstrated in Fig. 2(a), the macroscopic mechanical behavior during compression in the $1^{\text {st }}$ cycle is different from the other cycles. Comparing the work-hardening rate variation during compression in the $1^{\text {st }}$ cycle with the $2^{\text {nd }}$ cycle in Fig. 3(b), it is obvious that the constant work-hardening rate and the work-hardening rate increase regions were expanded, but the work-hardening rate decrease region almost disappeared in the $2^{\text {nd }}$ fatigue cycle. This trend can be explained by the neutron diffraction results in Fig. 4 that more grains involved in the tensile twinning deformation in the $2^{\text {nd }}$ tension than the initial tension in the $1^{\text {st }}$ cycle, which produced more twinned grains that were applicable for the detwinning process in the $2^{\text {nd }}$ fatigue cycle. Consequently, the detwinning 
process was prolonged, which was responsible for the expansion of the constant work-hardening rate region. It also led to the postponement and expansion of the transition from detwinning to dislocation dominated deformation regions, corresponding to the rapid strain-hardening region. Moreover, in the transition region, the dislocation slip became more and more active with the increase of stress. At the beginning, the dislocation density increased dramatically. When the dislocation density increased to a certain level, the increase of the dislocation density became gradual. It results in the work-hardening rate increasing, and then decreasing in the $1^{\text {st }}$ cycle.

Approaching the failure, the sample exhibited different deformation behavior from the previous cycles. This tendency can be observed from the experimental results in the $70^{\text {th }}$ cycle right before it failed in the $71^{\text {st }}$ cycle. It is found that the work-hardening rate during tension abruptly increased when the net flow stress was around $39 \mathrm{MPa}$ in Fig. 3(a) and the decrease of the diffraction peak intensity of the (00.2) grain in the axial direction decelerated after $129 \mathrm{MPa}$ during tension in the $70^{\text {th }}$ cycle in Fig. 5(a). It suggests that by the end of the fatigue life, the tensile twinning deformation became less dominant, and dislocation slips were inclined to be active due to cyclic loading, although the sample was not fully twinned during tension. It is believed that the increased dislocation density leads to the strain hardening behavior. On the other hand, the strain hardening results in the stress increase at the same strain level, which, in turn, facilitates the activation of dislocation slips. Subsequently, the sudden increase of the workhardening rate stems from the activation of the massive dislocation slips. It is thought that the increase of the dislocation density during tension may contribute to the stress concentration at the weak spots, such as the grain and twin boundaries, inside the sample, which accounts for the fatigue failure since the sample fractured at $71^{\text {st }}$ cycle during tension. 
The deformation history has a significant impact on deformation mechanisms during cyclic loading. In our previous studies [20, 54], the real-time in-situ neutron diffraction measurements were employed to study the deformation dynamics and deformation mechanisms of a rolled AZ31B Mg alloy during strain-path changes at a relative large plastic strain amplitude, +/- 0.07. The different deformation modes were labeled in each deformation stage, according to the work-hardening rate variations and neutron diffraction results. It was concluded that the work-hardening rate did not vary during the twinning and detwinning dominated deformation, and the rapid strain-hardening region was controlled by the transition from the twinning/detwinning to the dislocation dominated deformation, which agrees with the experimental observation in the present study. Besides that, it is found that the dislocation dominated deformation only existed during compression in the first two fatigue cycle in the current research. Since the initial tensile strain in the $1^{\text {st }}$ cycle was only 0.02 , which is smaller than the following fatigue cycle around 0.033 in Fig. 6(a), the population of twin grains after the initial tension in the $1^{\text {st }}$ cycle is much less than the other cycles. Consequently, the detwinning process was completed faster in the $1^{\text {st }}$ cycle than the following cycles, due to the inadequate amount of twinned grains. Once the detwinning process was exhausted, the further plastic deformation was governed by the dislocation slips, since no obvious diffraction peak intensity changes in the (00.2) and (10.0) grains were observed in the stress range 3 during compression in the $1^{\text {st }}$ cycle, as demonstrated in Fig. 5(c) and (d). However, the dislocation dominated deformation mode disappeared since the $5^{\text {th }}$ cycle, because the simultaneous $(00.2)$ peak intensity increase and (10.0) peak intensity decrease can be noticed in the entire plastic deformation region during compression in Fig. 5(c) and (d). As mentioned in Section 4.2, more and more grains were involved in the tensile twinning dominated deformation during tension with the 
increase of fatigue cycles. Subsequently, the amount of twinned grains is large enough after strain cycling, which yields that the detwinning process was not finished after the detwinning dominated deformation region and remained active in the rapid strain hardening region. Therefore, with the increase of fatigue cycles, the deformation modes in the plastic deformation region during compression changed from detwinning - transition - dislocation dominated deformation in the first two fatigue cycles to detwinning - transition dominated deformation in the following cycles. 


\section{Conclusions}

The real-time in-situ neutron diffraction has been taken advantage in the current study to investigate deformation mechanisms of a rolled AZ31B Mg alloy during the fully-reversed strain-controlled low-cycle fatigue. The relationship between the macroscopic low-cycle fatigue behavior and microscopic response at the grain level has been successfully established. Major conclusions can be drawn based on the present research, as described below:

(1) With the increase of fatigue cycles, more grains are involved in the twinning and detwinning process.

(2) The residual twins appear in the early fatigue life, and are responsible for the macroscopic cyclic hardening behavior.

(3) The early exhaustion of the detwinning process during compression is ascribed to the asymmetry of the stress-strain hysteresis loop.

(4) The compressive residual strain developed in each selected fatigue cycle results in the increase of the CRSS and the postponement of the onset of the tensile twinning.

(5) Right before the sample fractured, the tensile twinning became less dominant and dislocation slips turn out to be active in tension, although the sample was not fully twinned. The stress concentration at weak locations caused by the increase of the dislocation density is thought to be the main reason for the fatigue failure.

(6) The deformation history has significant impacts on the deformation modes during cyclic loading.

(7) The experimental results from the current study improve the fundamental understanding of the deformation dynamics and mechanisms under cyclic loading, which could benefit 
the development of theoretical modeling to describe the low-cycle fatigue behavior in HCP-structured polycrystalline materials 


\section{Acknowledgement}

The neutron work was carried out at the Spallation Neutron Source (SNS), Oak Ridge National Laboratory (ORNL), supported by the U.S. Department of Energy, Basic Energy Sciences, Scientific User Facilities Division. W.W. is supported by Columbus McKinnon Corporation and a Laboratory Directed Research and Development (LDRD) project of ORNL. PKL very much appreciates the financial support from the US National Science Foundation (DMR-0909037, CMMI-0900271, and CMMI-1100080) with C. Huber, C. V. Cooper, D. Finotello, A. Ardell, and E. Taleff as contract monitors, and DOE, Office of Fossil Energy, National Energy Technology Laboratory (DE-FE-0008855 and DE-FE-001194), with Mr. V. Cedro and S. Markovich as program managers. 


\section{References}

[1] Polmear IJ. Mater Sci Tech 1994;10:1.

[2] Aghion E, Bronfin B. Magnesium alloys development towards the 21(st) century. In: Kojima Y, Aizawa T, Kamado S, editors. Magnesium Alloys 2000, vol. 350-3. ZurichUetikon: Trans Tech Publications Ltd, 2000. p.19.

[3] Mordike B, Ebert T. Mater Sci Eng A 2001:37.

[4] Agnew S, Nie J. Scripta Mater 2010:671.

[5] Potzies C, Kainer KU. Adv Eng Mater 2004;6:281.

[6] Lee SG, Patel GR, Gokhale AM. Scripta Mater 2005;52:1063.

[7] Begum S, Chen DL, Xu S, Luo AA. Int J Fatigue 2009;31:726.

[8] Park SH, Hong SG, Lee BH, Bang W, Lee CS. Int J Fatigue 2010;32:1835.

[9] Begum S, Chen DL, Xu S, Luo AA. Metall Mater Trans A 2008;39A:3014.

[10] Yang F, Yin SM, Li SX, Zhang ZF. Mater Sci Eng A 2008;491:131.

[11] Agnew SR, Duygulu O. Int J Plast 2005;21:1161.

[12] Brown DW, Agnew SR, Bourke MAM, Holden TM, Vogel SC, Tome CN. Mater Sci Eng A 2005;399:1.

[13] Lou XY, Li M, Boger RK, Agnew SR, Wagoner RH. Int J Plast 2007;23:44.

[14] Agnew S, Yoo M, Tome C. Acta Mater 2001:4277.

[15] Barnett MR. Mater Sci Eng A 2007;464:1.

[16] Agnew SR, Tome CN, Brown DW, Holden TM, Vogel SC. Scripta Mater 2003;48:1003.

[17] Wu L, Jain A, Brown DW, Stoica GM, Agnew SR, Clausen B, Fielden DE, Liaw PK. Acta Mater 2008;56:688.

[18] Wu L, Agnew SR, Brown DW, Stoica GM, Clausen B, Jain A, Fielden DE, Liaw PK. Acta Mater 2008;56:3699.

[19] Wang YN, Huang JC. Acta Mater 2007;55:897.

[20] Wu W, An K, Huang L, Lee SY, Liaw PK. Scripta Mater 2013;69:358.

[21] Morrow B, McCabe R, Cerreta E, Tomé C. Metall Mater Trans A 2013:1.

[22] Proust G, Tome CN, Jain A, Agnew SR. Int J Plast 2009;25:861.

[23] Chen LJ, Wang CY, Wu W, Liu Z, Stoica GM, Wu L, Liaw PK. Metall Mater Trans A 2007;38A:2235.

[24] Li QZ, Yu Q, Zhang JX, Jiang YY. Scripta Mater 2010;62:778.

[25] Chen C, Liu TM, Lv CL, Lu LW, Luo DZ. Mater Sci Eng A 2012;539:223.

[26] Lv F, Yang F, Li SX, Zhang ZF. Scripta Mater 2011;65:53.

[27] Zhang J, Yu Q, Jiang Y, Li Q. Int J Plast 2011;27:768.

[28] Yu Q, Zhang J, Jiang Y, Li Q. Int J Fatigue 2012;44:225.

[29] Begum S, Chen DL, Xu S, Luo AA. Mater Sci Eng A 2009;517:334.

[30] Li Q, Yu Q, Zhang J, Jiang Y. Scripta Mater 2011;64:233.

[31] Guillemer C, Clavel M, Cailletaud G. Int J Plast 2011;27:2068.

[32] Tsushida M, Shikada K, Kitahara H, Ando S, Tonda H. Mater Trans 2008;49:1157.

[33] Liu WC, Wu GH, Zhai CQ, Ding WJ, Korsunsky AM. Int J Plast 2013;49:16.

[34] Yin SM, Li SX. J. Mater. Sci. Technol. 2013;29:775.

[35] Mirza FA, Chen DL, Li DJ, Zeng XQ. Mater Sci Eng A 2013;575:65.

[36] Zhu R, Ji WQ, Wu YJ, Cai XT, Yu Y. Mater Des 2012;41:203.

[37] Sajuri ZB, Miyashita Y, Mutoh Y. Fatigue Fract Eng Mater Struct 2005;28:373.

[38] Bhuiyan MS, Mutoh Y, Murai T, Iwakami S. Int J Fatigue 2008;30:1756.

[39] Wu L, Agnew SR, Ren Y, Brown DW, Clausen B, Stoica GM, Wenk HR, Liaw PK. Mater 
Sci Eng A 2010;527:7057.

[40] Park SH, Hong SG, Bang W, Lee CS. Mater Sci Eng A 2010;527:417.

[41] Lv F, Yang F, Duan QQ, Yang YS, Wu SD, Li SX, Zhang ZF. Int J Fatigue 2011;33:672.

[42] Jordon JB, Brown HR, El Kadiri H, Kistler HM, Lett RL, Baird JC, Luo AA. Int J Fatigue 2013;51:8.

[43] Neil CJ, Agnew SR. Int J Plast 2009;25:379.

[44] Choi SH, Kim DW, Seong BS, Rollett AD. Int J Plast 2011;27:1702.

[45] Wang H, Wu PD, Wang J, Tome CN. Int J Plast 2013;49:36.

[46] Wang H, Wu PD, Wang J. Int J Plast 2013;47:49.

[47] Guo XQ, Wu W, Wu PD, Qiao H, An K, Liaw PK. Scripta Mater 2013;69:319.

[48] Lin XZ, Chen DL. Mater Sci Eng A 2008;496:106.

[49] Yu Q, Zhang JX, Jiang YY, Li QZ. Int J Fatigue 2012;36:47.

[50] Yin SM, Yang HJ, Li SX, Wu SD, Yang F. Scripta Mater 2008;58:751.

[51] Yu Q, Qi L, Chen K, Mishra RK, Li J, Minor AM. Nano Lett 2012;12:887.

[52] Brown DW, Jain A, Agnew SR, Clausen B. Twinning and detwinning during cyclic deformation of Mg alloy AZ31B. In: Chandra T, Tsuzaki K, Militzer M, Ravindran C, editors. Thermec 2006, Pts 1-5, vol. 539-543. Stafa-Zurich: Trans Tech Publications Ltd, 2007. p.3407.

[53] Lee SY, Wang H, Gharghouri MA, Nayyeri G, Woo W, Shin E, Wu PD, Poole WJ, Wu W, An K. Acta Mater 2014;73:139.

[54] Wu W, Qiao H, An K, Guo X, Wu P, Liaw PK. Int J Plast 2014;62:105.

[55] Wu W, Lee SY, Paradowska AM, Gao YF, Liaw PK. Mater Sci Eng A 2012;556:278.

[56] Wang XL, Holden TM, Rennich GQ, Stoica AD, Liaw PK, Choo H, Hubbard CR. Physica B 2006;385:673.

[57] Wang XL, Holden TM, Stoica AD, An K, Skorpenske HD, Jones AB, Rennich GQ, Iverson EB. Mechanical Stress Evaluation by Neutrons and Synchrotron Radiation 2010;652:105.

[58] An K, Skorpenske HD, Stoica AD, Ma D, Wang XL, Cakmak E. Metall Mater Trans A 2011;42A:95.

[59] Cai L, Liu ZC, An K, Liang CD. J Mater Chem A 2013;1:6908.

[60] Cai L, An K, Feng ZL, Liang CD, Harris SJ. J Power Sources 2013;236:163.

[61] Chen Y, Yang L, Ren F, An K. Sci Rep 2014;4.

[62] Yu D, An K, Chen Y, Chen X. Scripta Mater 2014;89:45.

[63] An K. ORNL Report No. ORNL-TM-2012-621 2012;Oak Ridge National Laboratory, Oak Ridge, TN.

[64] Gharghouri MA, Weatherly GC, Embury JD, Root J Philos Mag A 1999;79:1671.

[65] Agnew SR, Yoo MH, Tome CN. Acta Mater 2001;49:4277. 


\section{Figure Captions}

Figure 1. The two-dimensional (2-D) and three-dimensional (3-D) real-time in-situ neutron diffraction contour results during low-cycle fatigue experiment for: (a) the 2-D patterns in the axial direction, (b) the 2-D patterns in the radial direction, (c) the 3-D patterns in the axial direction, and (d) the 3-D patterns in the radial direction.

Figure 2. (a) The hysteresis loops at different fatigue cycles under a total strain amplitude of +/- 0.02 for the ND sample. (b) The peak stresses, stresses at zero strains, and mean stresses variation during low-cycle fatigue experiment. (c) The $0.2 \%$ proof stress evolution as a function of number of cycles. (d) The plastic strain as a function of number of cycles.

Figure 3. The work-hardening rate $(\theta)$ as a function of net flow stress $\left(\sigma-\sigma_{0.2}\right)$ at different fatigue cycles: (a) tension and (b) compression. The slopes of the work-hardening rate vs. net flow stress curve in the $1^{\text {st }}$ and $70^{\text {th }}$ cycle are indicated as black and purple dash-dot lines, respectively. The letters and numbers are marked on the dash-dot lines to represent the different work-hardening behaviors.

Figure 4. The real-time in-situ neutron diffraction peak intensity evolution at different fatigue cycles: (a) and (b) the diffraction peak intensity variation in the axial and radial directions, respectively. The mechanical stress evolution in each fatigue cycle is plotted on the secondary $\mathrm{Y}$ axis as open square symbols in each figure.

Figure 5. The real-time in-situ neutron diffraction peak intensity evolution in the axial direction at different fatigue cycles: (a) and (b) the (00.2) and (10.0) peaks during tension, respectively, (c) and (d) the (00.2) and (10.0) peaks during compression, respectively. The slopes of the (00.2) diffraction peak intensity vs. engineering stress curve in the $1^{\text {st }}$ and $70^{\text {th }}$ cycle are specified as black and purple dash-dot lines, respectively. The letters and numbers are adopted from Fig. 3(b) for the different work-hardening behaviors.

Figure 6. (a) The stress-strain hysteresis loop of the $50^{\text {th }}$ fatigue cycle. (b) The peak stresses, $0.2 \%$ proof stresses, and stresses at dividing points as a function of fatigue cycles.

Figure 7. The critical resolved shear stress (CRSS) variation at different fatigue cycles. The residual lattice strains of (00.2) grains in the axial direction after compression as a function of fatigue cycles are plotted on the secondary $\mathrm{Y}$ axis. 

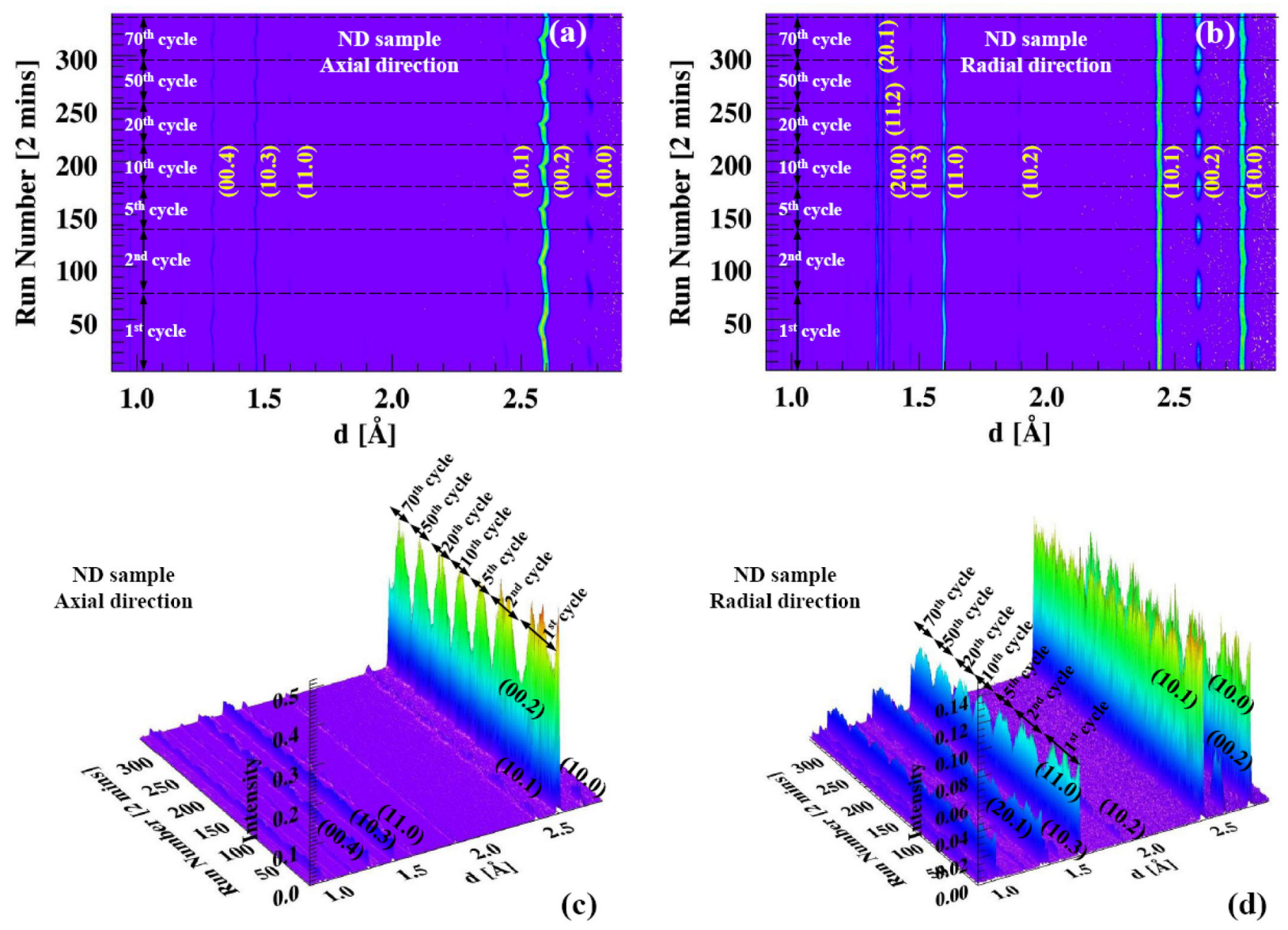

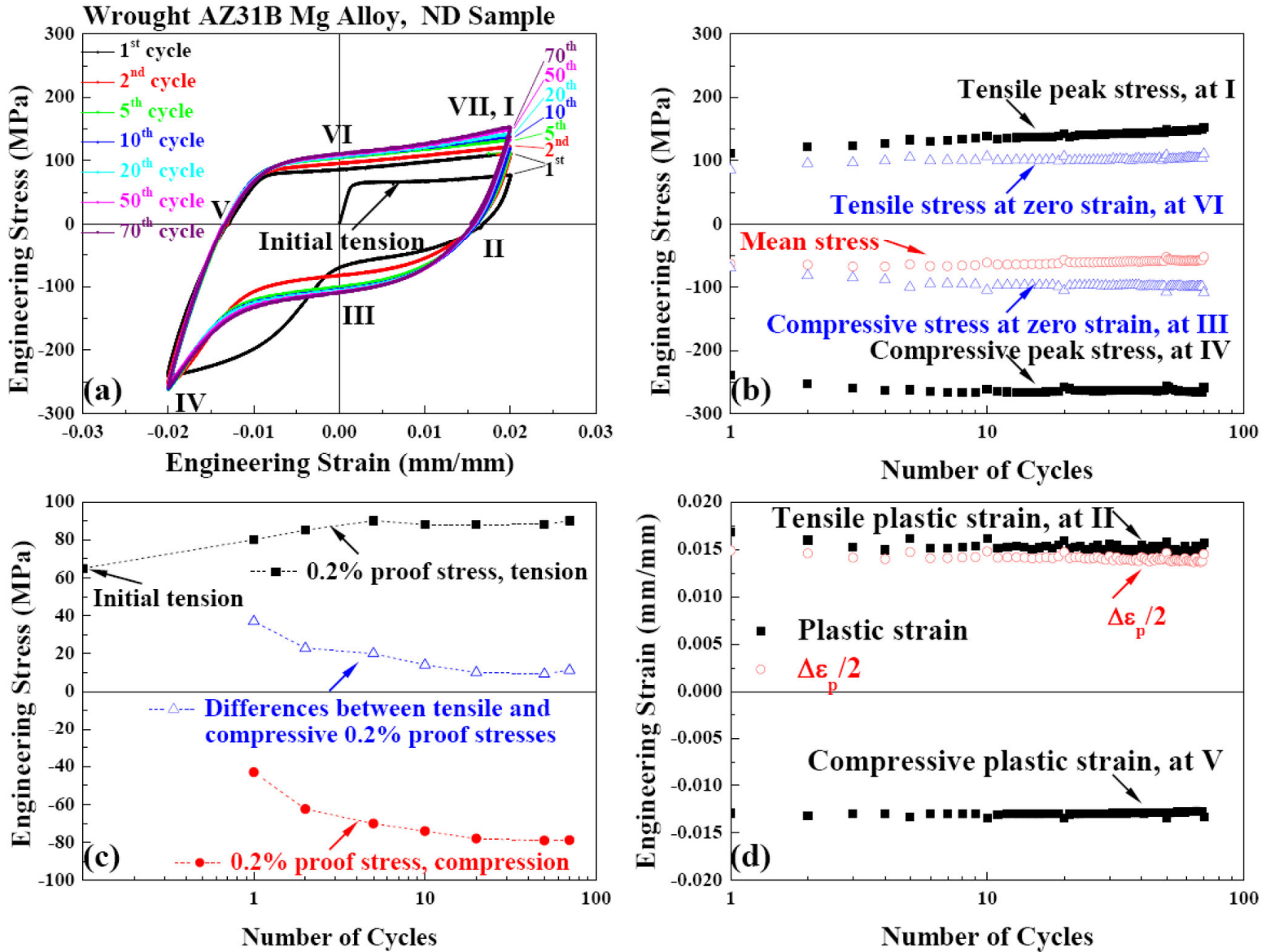

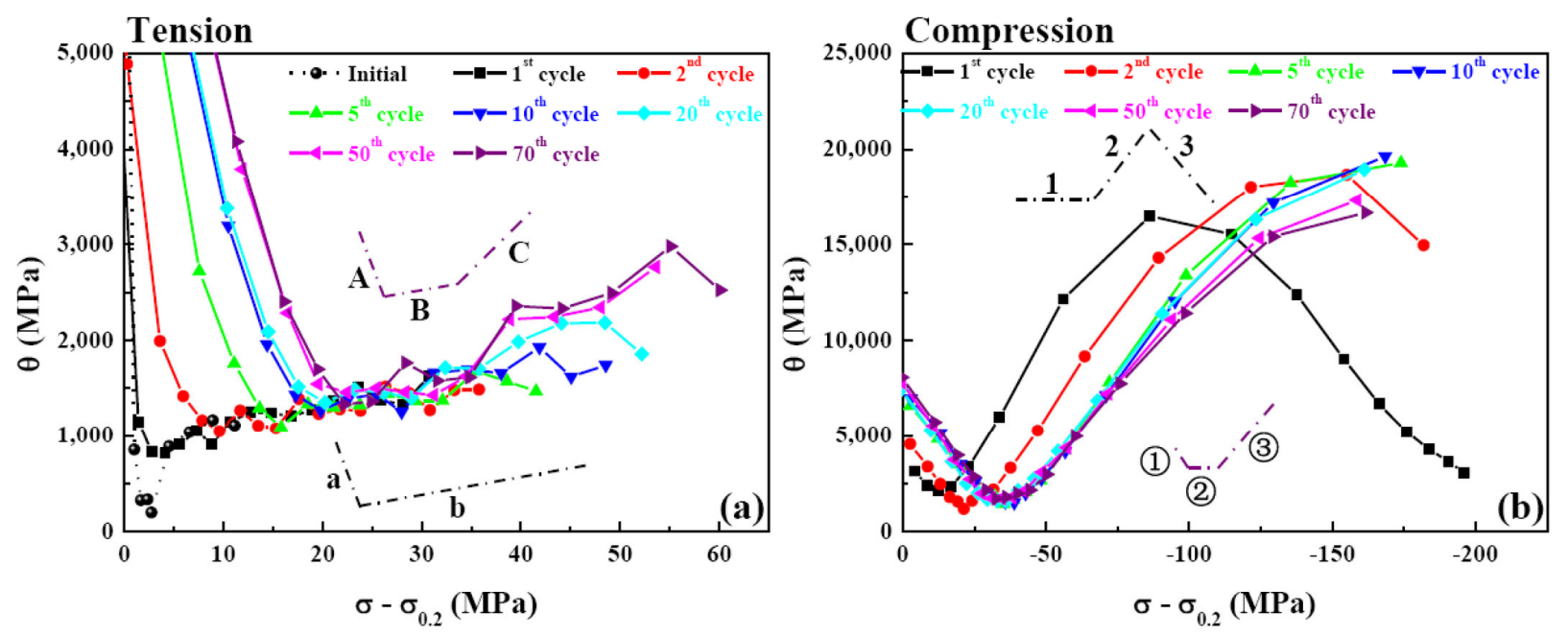

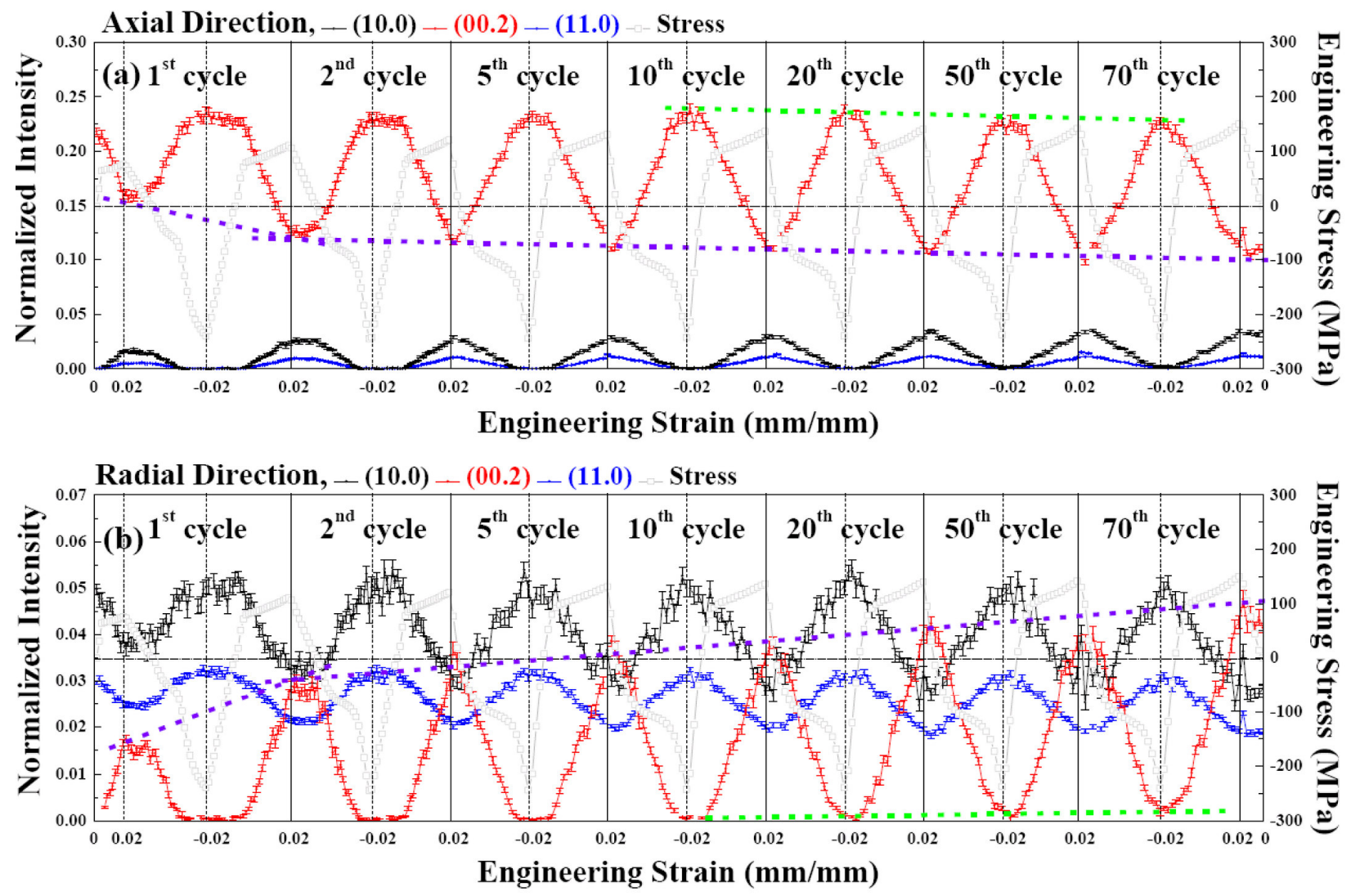

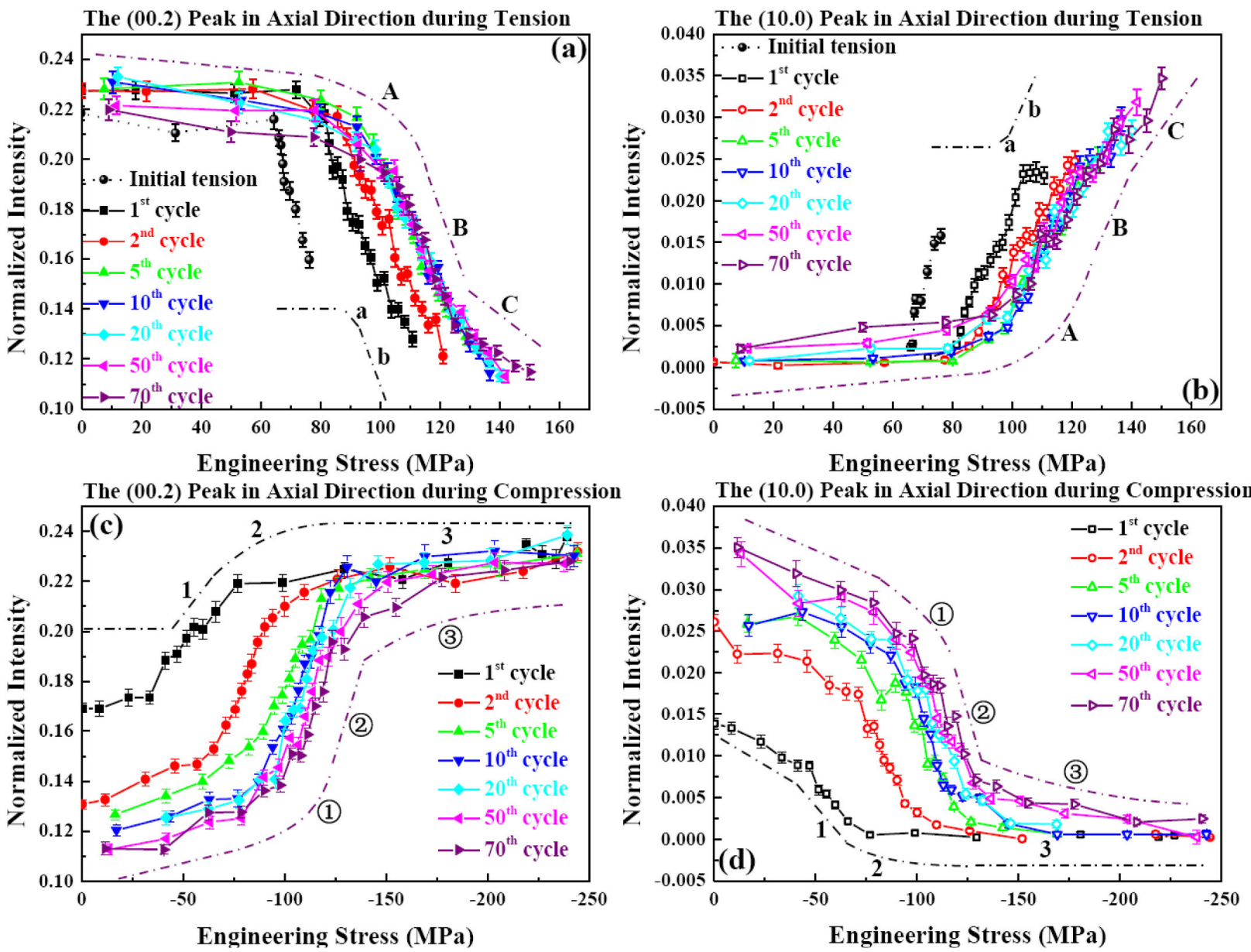

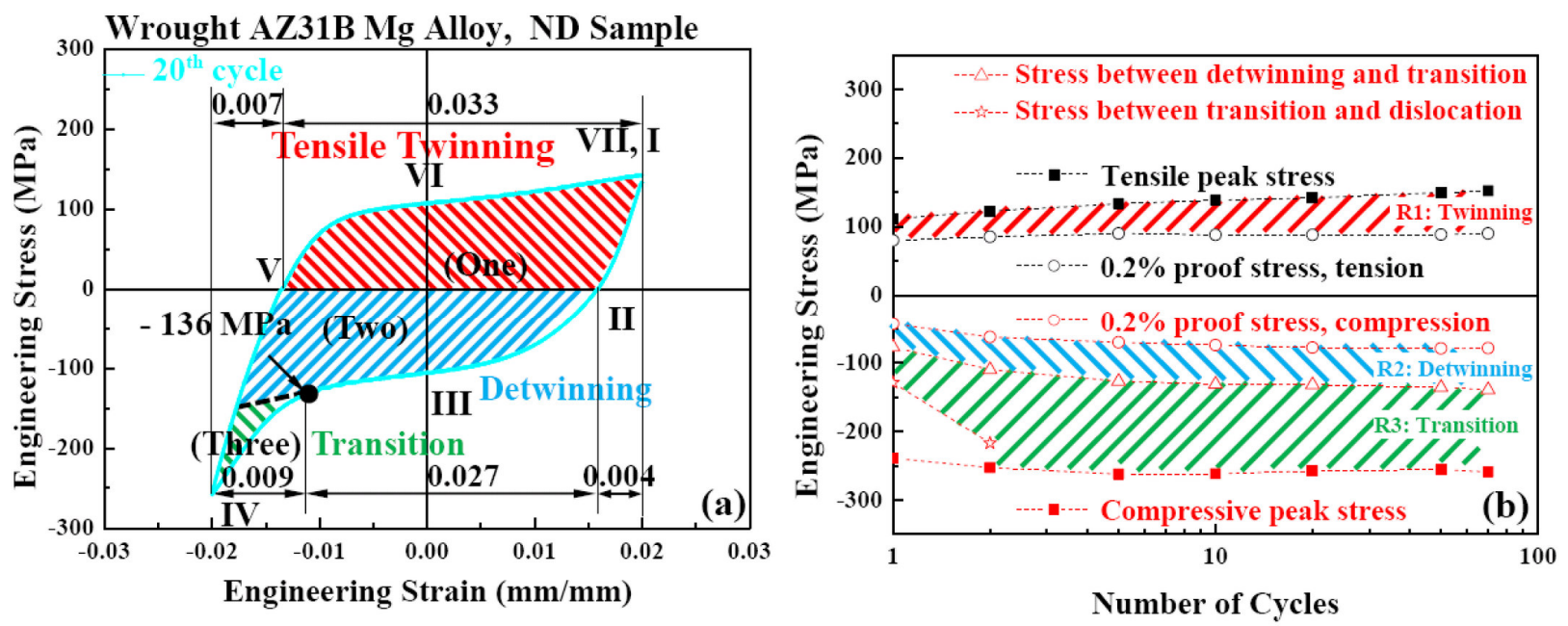


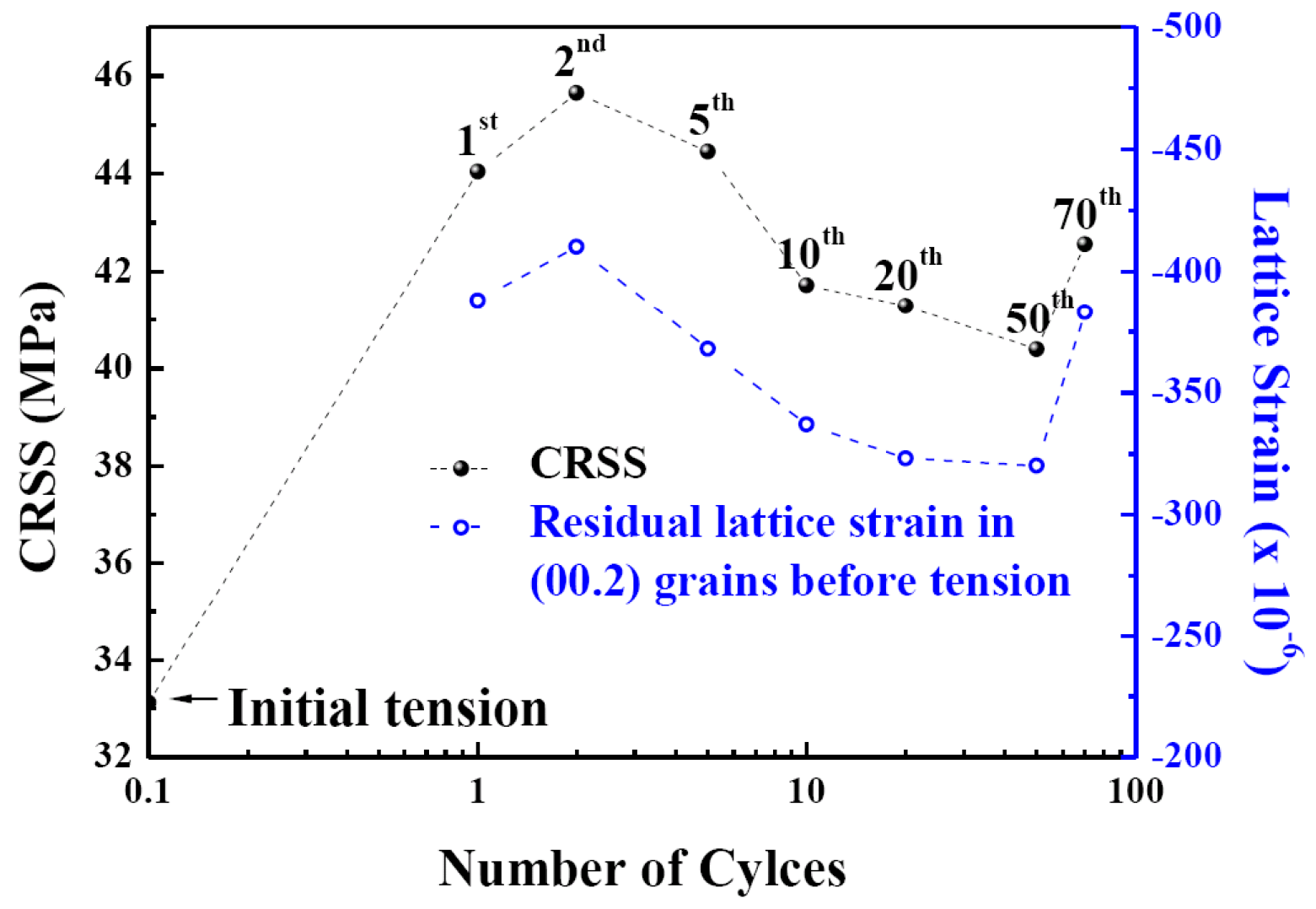

